\title{
Synthesis of Porous $\mathrm{SiO}_{2}$ Spherical Particles by Sol-Gel Methods in W/O Emulsion
}

\author{
Akiko Hirose, Hiroshi Yamashita and Takashi MaEkawa \\ Department of Applied Chemistry, Faculty of Engineering, Ehime University, \\ Matsuyama 790, Japan
}

\begin{abstract}
Dispersed porous spherical silica particles were prepared by a sol-gel process from solutions of tetramethoxysilane (TMOS), polyethyleneglycol (PEG), $\mathrm{H}_{2} \mathrm{O}$ and organic solvent in $\mathrm{W} / \mathrm{O}$ (water in oil phase) emulsions. The interconnected pores can be made by eliminating PEG from gel bodies. Such PEG originates by phase separation between the PEG and the polymerizing silica in common solvents. Microstructures of the particles are affected by the compositions of the starting solutions. The pore diameter increases with an increase in molecular weight of PEG when the molar ratio of PEG as monomer unit to TMOS is fixed to 0.3. The pore diameter decreases and specific surface area increases with an increase in the content of PEG having molecular weight of 50000 when the [PEG]/[TMOS] molar ratio is greater than 0.3 . The pore diameter decreases by use of basic solvents such as $N, N$-dimethylformamide (DMF) and formamide (FA) instead of methanol.
\end{abstract}

Keywords Porous silica, sol-gel method, high-performance liquid chromatography, packing material, W/O emulsion

Surface modified silica gel particles have been used as packing materials for the high-performance liquid chromatography (HPLC). The control of pore sizes and particle sizes of the gels is one of the important problems in this field. Generally, pores of commercial particles consist of interstices of secondary silica particles. For better chemical stability and mechanical strength, however, glasses of interconnected pore structure are preferred. In a previous paper ${ }^{1}$, we developed a new type of porous silica particles by thermal and successive acid treatments of sodium borosilicate glasses prepared by the sol-gel process. Although the spherical porous glasses of interconnected pore structure were obtained, it required two steps: first thermal, then acid treatment.

Nakanishi et al. ${ }^{2-5}$ reported methods for preparing monolithic porous silica gels by the sol-gel process; thus, porous silica gels were prepared from solutions of tetraethoxysilane (TEOS) containing polymers such as polyacrylic acid (PAA). They differentiated the type of morphology and average domain size in terms of the concentration, molecular weight of PAA and solubility parameter of the chemical additives. Ota et al. ${ }^{6}$ synthesized organic-inorganic composite gels from tetraethoxysilane-polyethyleneglycol (PEG)- $\mathrm{H}_{2} \mathrm{O}$-methanol $(\mathrm{MeOH})$. Uo et al. $^{7}$ succeeded in preparing porous silica gels from solutions of tetramethoxysilane (TMOS)-PEG- $\mathrm{H}_{2} \mathrm{O}-\mathrm{MeOH}$. The pore diameters decreased with increases of concentration and molecular weight of PEG. In the initial solution, immiscibility can not be observed. However, progress of polymerization of TMOS shifts the critical solution temperature to a higher temperature and the solutions are brought into a two-phase region, i.e. one phase of PEG+solvent and another phase of polymerizing silicatsolvent. This type of phase separation differs from that seen when the temperature of the solution is lowered below the critical solution temperature. The type (spinodal or nucreation and growth) and the degree of phase separation depend on when sol-gel transition occurs. Thus, the factors which determine the morphology of pore structure are mutual solubility of PEG and some organic solvent, molecular weight of PEG and rates of hydrolysis and condensation of TMOS. In this paper, a procedure to prepare spherical silica particles of interconnected pore structure by using the sol-gel reaction in $\mathrm{W} / \mathrm{O}$ emulsion is developed and the optimum conditions are examined.

\section{Experimental}

\section{Syntheses of porous silica particles in $W / O$ emulsions}

The compositions of starting solutions are shown in Table 1. Here, the molar ratios of PEG are expressed by monomer units. The particles made using PEG with molecular weights of 50000 and 20000 are denoted hereafter as $\mathrm{SiO}_{2}-5$ and $\mathrm{SiO}_{2}-2$, respectively. PEG having molecular weights of $2000000,500000,2000$ and 200 were also used. Hydrochloric acid was used as a catalyst for hydrolysis. Distilled water containing PEG and hydrochloric acid was spouted slowly through a glass capillary into $2.5 \mathrm{~g}$ TMOS diluted with $\mathrm{MeOH}$ at room temperature during $45 \mathrm{~min}$. The mixed solution was stirred vigorously during an additional $45 \mathrm{~min}$ to $3 \mathrm{~h}$ in a beaker 
Table 1 Composition of starting solutions

\begin{tabular}{lccccc}
\hline & \multicolumn{5}{c}{ Molar ratio } \\
\cline { 2 - 6 } & TMOS & $\mathrm{H}_{2} \mathrm{O}$ & $\mathrm{HCl}$ & $\mathrm{CH}_{3} \mathrm{OH}$ & PEG \\
\hline Series A & 1 & 20 & 0.01 & 9 & $0.1-1.0$ \\
Series B & 1 & 20 & 0.01 & 9 & $0.1-1.0$ \\
Series C & 1 & 20 & 0.01 & $0-12$ & 0.3 \\
\hline
\end{tabular}

PEG molecular weights of series A, B and C are 50000, 20000 and 50000 respectively.

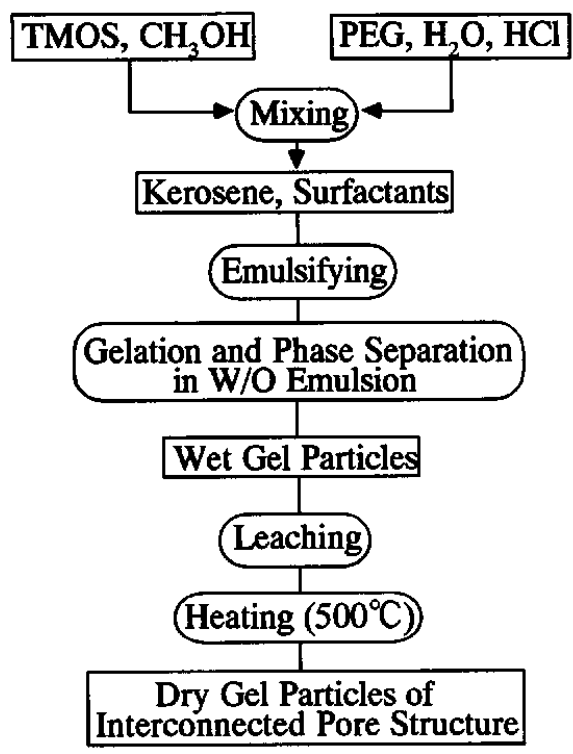

Fig. 1 Preparation of porous $\mathrm{SiO}_{2}$ spherical particles.

covered with aluminum foil, as shown in Fig. 1. Then the sol solution was poured into a $100 \mathrm{ml}$ Teflon beaker containing $26.1 \mathrm{~g}$ of kerosene and $30 \mathrm{wt} \%$ of surfactants (the weight ratio of sorbitan monooleate: sorbitan monostearate is $3: 1$ ). The resulting solution was agitated uniformly using a homogenizer (Tokushu Kika Kogyo Co., Homomixer M) at a rate of $2000 \mathrm{rpm}$ at $45^{\circ} \mathrm{C}$; thus, the hydrolysis and condensation reaction is as shown in Eqs. (1) - (3),

$$
\begin{aligned}
& \mathrm{Si}\left(\mathrm{OCH}_{3}\right)_{4}+n \mathrm{H}_{2} \mathrm{O} \longrightarrow \\
& \quad \mathrm{Si}\left(\mathrm{OCH}_{3}\right)_{4-n}(\mathrm{OH})_{n}+n \mathrm{CH}_{3} \mathrm{OH} \\
& -\mathrm{Si}(\mathrm{OH})+-\mathrm{Si}(\mathrm{OH}) \longrightarrow-\mathrm{Si}-\mathrm{O}-\mathrm{Si}-+\mathrm{H}_{2} \mathrm{O} \\
& -\mathrm{Si}\left(\mathrm{OCH}_{3}\right)+-\mathrm{Si}(\mathrm{OH}) \longrightarrow-\mathrm{Si}-\mathrm{O}-\mathrm{Si}-+\mathrm{CH}_{3} \mathrm{OH}
\end{aligned}
$$

were again continued in the water phase of $\mathrm{W} / \mathrm{O}$ emulsion. After gelation (usually $30 \mathrm{~min}$ after emulsifying), particles precipitated at the bottom. These wet particles were filtrated, washed with hexane and acetone, and dried for $3 \mathrm{~d}$ in an oven at $40^{\circ} \mathrm{C}$. A preliminary thermal analysis of the particles by DTA showed an exothermic

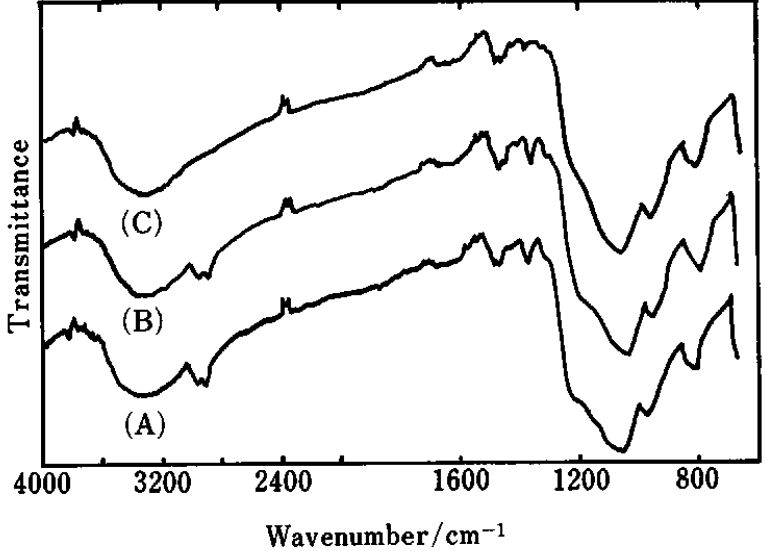

Fig. 2 FT-IR spectra of $\mathrm{SiO}_{2}$ particles. Heat treatment temperature: A, $40^{\circ} \mathrm{C} ; \mathrm{B}, 200^{\circ} \mathrm{C} ; \mathrm{C}, 500^{\circ} \mathrm{C}$.

peak around $370^{\circ} \mathrm{C}$ due to burning of the residual organic solvents, including PEG. FT-IR spectra of particles heated at various temperatures are shown in Fig. 2. Here an absorption band at around $3400 \mathrm{~cm}^{-1}$ is assigned to $\mathrm{Si}-\mathrm{OH}$ stretching vibration. An absorption around $2900 \mathrm{~cm}^{-1}$ band assigned to PEG $\left(-\mathrm{CH}_{2} \mathrm{CH}_{2-}\right.$ stretching vibration) disappeared at $500^{\circ} \mathrm{C}$. Thus, the temperature was raised to $200^{\circ} \mathrm{C}$ at a rate of $5^{\circ} \mathrm{C} / \mathrm{h}$ and kept at this temperature for $5 \mathrm{~h}$. Finally the temperature was raised to $500^{\circ} \mathrm{C}$ at a rate of $50^{\circ} \mathrm{C} / \mathrm{h}$ and was kept for $12 \mathrm{~h}$ in an electric furnace.

\section{Characterization of the particles}

The specific surface area and pore size distribution were measured using an analyzer (Shibata Co., SA-1100) and a poresizer (Micromeritix Co., 9303), respectively. The particle shape and particle size distribution were determined from images of a scanning electron microscope (Hitachi Co., S-510).

\section{Results and Discussion}

Effect of molecular weight and concentration of PEG on morphology of the particles

When PEG of molecular weights of 200 and 2000 were used, the interconnected pore structure did not form; thus, the particles consisted of aggregations of secondary particles of $\mathrm{SiO}_{2}$. The pore diameter and specific surface area were $4 \mathrm{~nm}$ and $500 \mathrm{~m}^{2} / \mathrm{g}$; such values are seen in silica gels prepared by the usual sol-gel method without any organic macromolecules. On the other hand, when PEG having molecular weights of 500000 and 2000000 were used, it was difficult to make spherical particles in $\mathrm{W} / \mathrm{O}$ emulsion due to the high viscosity of the solutions. Figure 3 shows an typical particle size distribution of $\mathrm{SiO}_{2}-5$ under the present experimental conditions. They are well dispersed and the average particle diameter was about $15 \mu \mathrm{m}$. Dependences of the pore size (central values of pore size distribution curves) and specific surface area of $\mathrm{SiO}_{2}-5$ and $\mathrm{SiO}_{2}-2$ particles on the 


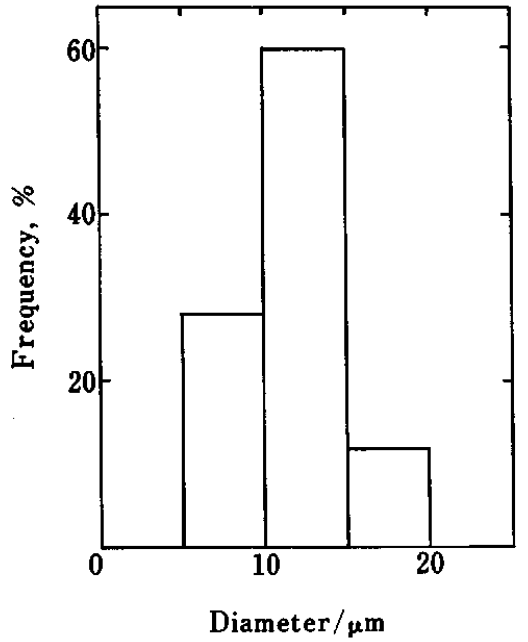

Fig. 3 Typical particle size distribution of $\mathrm{SiO}_{2}-5$.

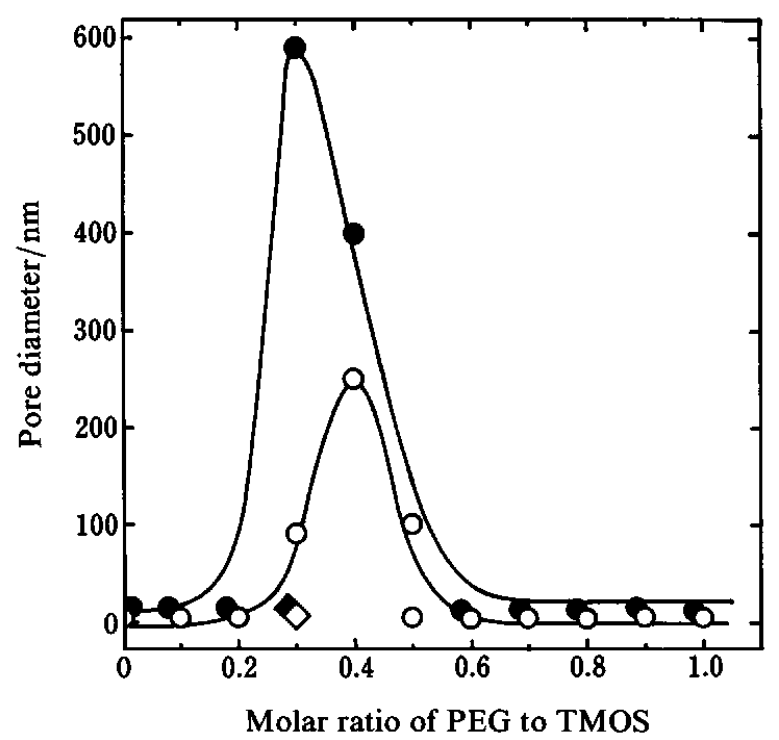

Fig. 4 Dependences of pore size on the concentration of PEG. $\diamond:$ PEG-200; $\diamond$ PEG-2000; O: PEG-20000; PEG-50000.

concentration of PEG are shown in Figs. 4 and 5, respectively. The pore size decreased with an increase in the concentration of $P E G$ in $\mathrm{SiO}_{2}-5$ and $\mathrm{SiO}_{2}-2$ particles when the molar ratio of PEG to TMOS was greater than 0.3 or 0.4 , respectively. This tendencies are similar to the results of monolithic gels made by Uo et al. ${ }^{7}$ The specific surface area increased with an increase of the concentration of PEG, when the molar ratio of PEG to TMOS is also greater than 0.3 or 0.4 . Figure 6 represent morphologies of $\mathrm{SiO}_{2}-5$ particles when the [PEG]/ [TMOS] ratios are $0.2,0.3$ and 0.8 . The specific surface area of interconnected structure increased with an increase of molecular weight of PEG when the molar ratio of [PEG] to [TMOS] is fixed at 0.3 . The large pores were observed in particles when PEG of lower molecular weight were used. On the other hand, when

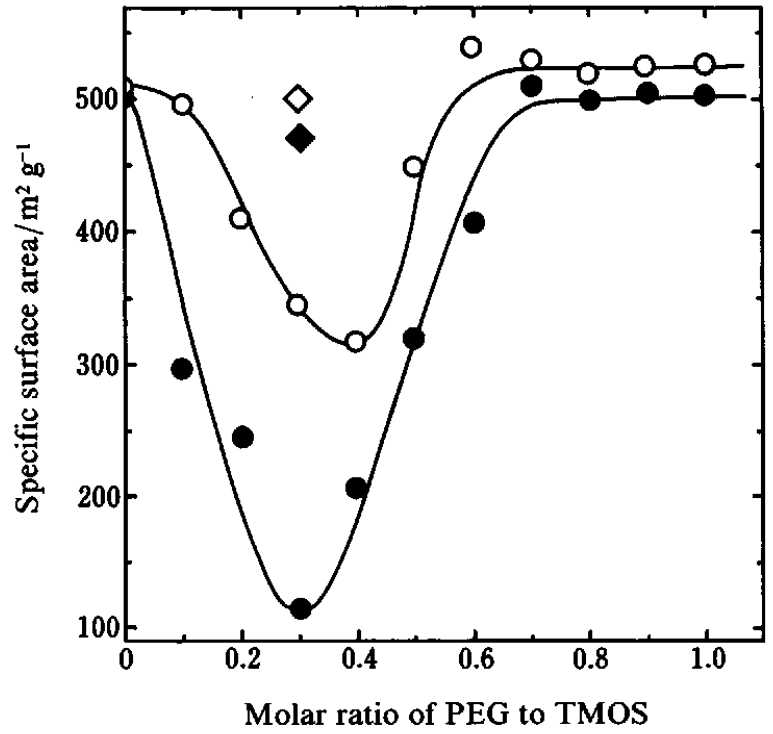

Fig. 5 Dependence of specific surface area on the concentration of PEG; symbols are the same as in Fig. 4.

the $[\mathrm{PEG}] /[\mathrm{TMOS}]$ ratio is lower than 0.2 , the particles consist of aggregations of small secondary particles, so that the specific surface area increases due to the interstices of these secondary particles.

\section{Effect of solvents on morphology of the particles}

Figure 7 shows an SEM image of the $\mathrm{SiO}_{2}-5$ particle when the molar ratio of PEG to TMOS is fixed to 0.3 and that of MeOH to TMOS is 6 or 12. Generally, an isolated pore structure was seen when the $[\mathrm{MeOH}] /$ [TMOS] molar ratio is smaller than 6 , which contrasts to that seen when this ratio is 9 (Fig. 6). On the other hand aggregation of silica secondary particles was seen in particles when the [MeOH] /[TMOS] ratio is 12. Additions of various organic solvents other than $\mathrm{MeOH}$ lead to the changes of the pore diameter of the resultant particles. In Table 2 the specific surface area and pore diameter of the $\mathrm{SiO}_{2}-5$ particles are listed; here ethyleneglycol (EG), trimethyleneglycol (TMG), $N, N$-dimethyl formamide (DMF) and formamide (FA), whose mole ratio to TMOS was fixed to 9, were used. Nakanishi et al. ${ }^{4}$ reported that the average domain size decreased with an increase of solubility parameter. Solubility parameters of EG, DMF and FA are 14.6, 12.1 and 19.2, so that a clear relation of pore sizes to solubility parameters cannot be seen. Rather, DMF and FA, which are classified as Lewis bases, seem to affect the diameters and pore sizes of the particles. The pore diameter obtained by using DMF seems larger than that obtained by FA. FA makes large pores in gels due to the formation of large primary-particles compared with those of DMF. ${ }^{8}$ The primary-particles distribute uniformly over the bulk gel. Therefore, the degree of coalescence among the particles is less than that obtained by using DMF in the hydrolysis and polymerization reactions of TMOS. ${ }^{1}$ 

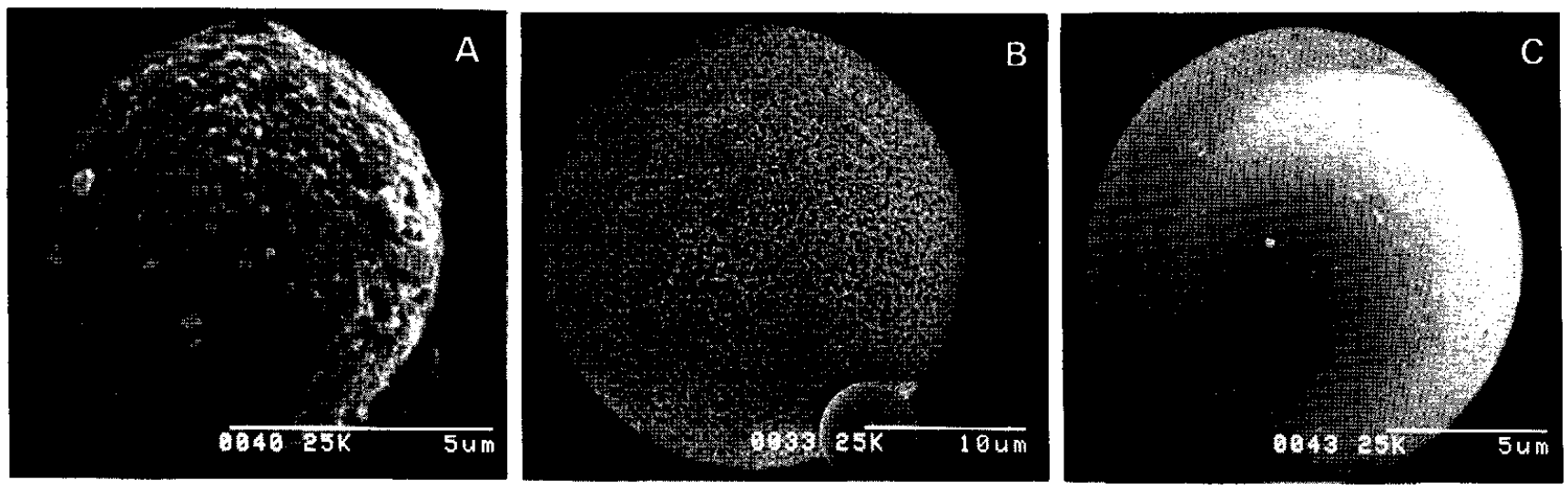

Fig. 6 Morphologies of $\mathrm{SiO}_{2}-5$ particles. [PEG]/[TMOS] ratio: $\mathrm{A}, 0.2 ; \mathrm{B}, 0.3 ; \mathrm{C}, 0.8$.
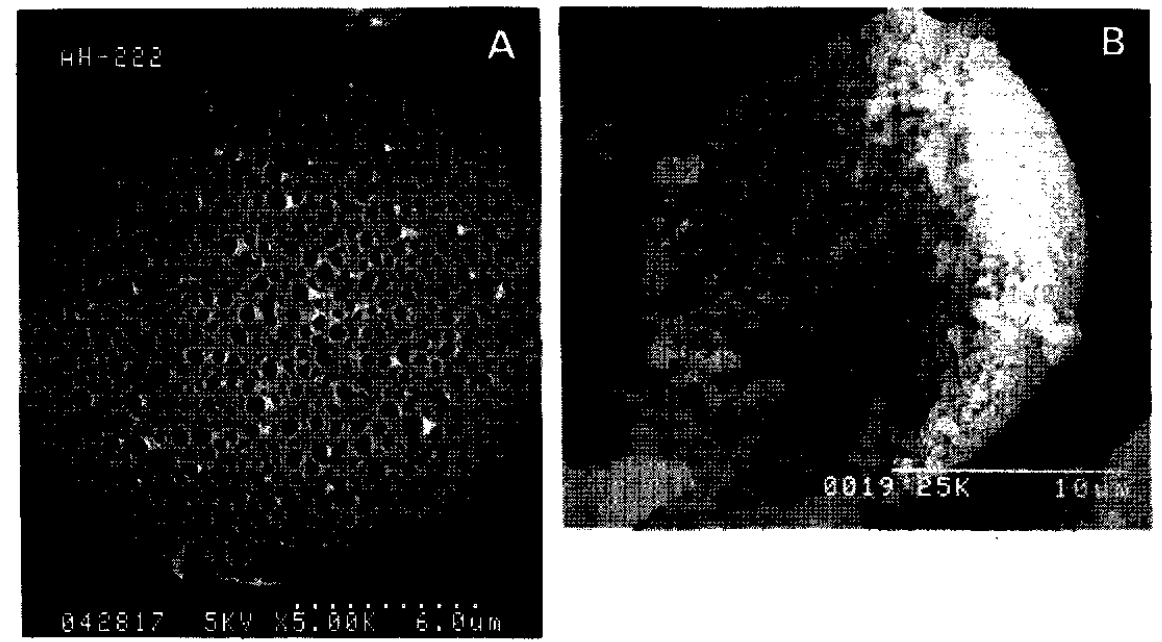

Fig. $7 \mathrm{SEM}$ image of the $\mathrm{SiO}_{2}-5$ particles. [PEG]/[TMOS] ratio is 0.3 . $[\mathrm{MeOH}] /[\mathrm{PEG}]$ ratio: $\mathrm{A}, 6 ; \mathrm{B}, 12$.

Table 2 Effect of solvents on specific surface area and pore diameter of $\mathrm{SiO}_{2}$ particles

\begin{tabular}{lcc}
\hline \multicolumn{1}{c}{ Solvent } & $\begin{array}{c}\text { Specific surface } \\
\text { area } / \mathrm{m}^{2} \mathrm{~g}^{-1}\end{array}$ & $\begin{array}{c}\text { Pore diameter/ } \\
\mathrm{nm}\end{array}$ \\
\hline Ethylene glycol & 112 & 590 \\
Methanol & 113 & 590 \\
Trimethylene glycol & 234 & 320 \\
$N, N$-Dimethyl formamide & 360 & 86 \\
Formamide & 377 & 60 \\
\hline
\end{tabular}

TMOS : solvent : $\mathrm{H}_{2} \mathrm{O}: \mathrm{HCl}: \mathrm{PEG}(50000)=1: 9: 20: 0.01: 0.3$.

\section{Time of hydrolysis}

In the present experiment, hydrolysis was pursued at room temperature between $45 \mathrm{~min}$ and $3 \mathrm{~h}$ after mixing of TMOS solution with PEG and then the temperature was changed to $45^{\circ} \mathrm{C}$ in W/O emulsions; here gelations occurred in comparatively short times $(30 \mathrm{~min})$. The pore sizes decreased with an increase in times of hydrolysis before emulsifying. For example, pore sizes are $590 \mathrm{~nm}$ and $300 \mathrm{~nm}$ when hydrolyses were performed for $45 \mathrm{~min}$ or for $90 \mathrm{~min}$ or more, respectively. With an increase in the hydrolysis time, the polymerization in $\mathrm{W} /$ $\mathrm{O}$ emulsion may proceed faster and PEG was removed out from the network of silica+PEG system in an emulsion to oil phase. The pore sizes decrease due to large volume fractions of the silica phase. DMF and FA may accelerate the rate of hydrolysis as catalysts.

\section{Mechanism of phase separation}

As shown in the literature ${ }^{5}$, schematic phase diagrams for the present system are also shown in Fig. 8. Before the start of the polymerization of TMOS, the solution consists of one phase under a common solvent such as methanol, shown in Fig. 8 (A); here the starting composition is denoted by an open circle. With progress of polymerization of hydrolyzed silica, the compatibility between silica and PEG in the presence of methanol is lowered and the upper critical solution temperature should shift to higher temperature (Fig. 8 (B)). The solution is brought into a two-phase region. When 


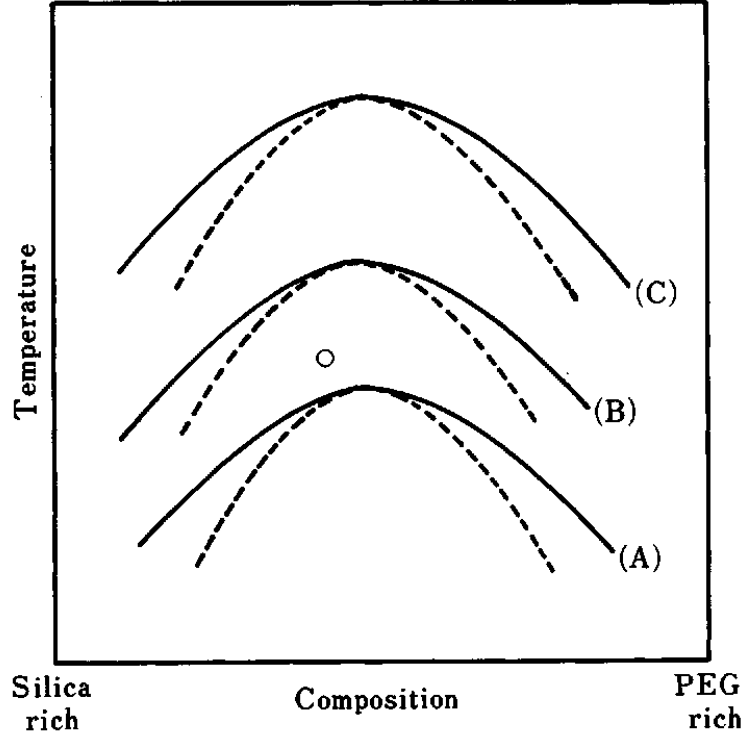

Fig. 8 Schematic shift of the binodal line (solid lines) and spinodal line (broken lines) with polymerization of TMOS. Starting composition is indicated by a small open circle. The polymerization of TMOS proceeds successively to (A), (B) and (C) (see text ${ }^{5}$.

gelation proceeds in a one-phase region, the resultant particles consist of aggregations of secondary particles of $\mathrm{SiO}_{2}$, as seen in Fig. 6(A), where the concentration of PEG is low and that of methanol is high. The polymerization proceeds in a similar way to that seen in an absence of PEG. On the other hand, when the concentration of methanol is low, PEG was included in a silica network, so that $\mathrm{SiO}_{2}$ particles with isolated pores were formed, due to elimination of PEG from the silica network by thermal treatment (Fig. 7(B)).

When gelation occurs in a two-phase region, the phase separation proceeds by a spinodal decomposition mechanism. The interconnected pore structure can be seen in Figs. 6(B) and 6(C). The pore size increased with an increase in the concentration of PEG. The relationship $^{9}$ between pore diameter or structural period $\left(\Lambda_{\mathrm{m}}\right)$ and spinodal temperature $\left(T_{\mathrm{s}}\right)$ is expressed by Eq. (4):

$$
\Lambda_{\mathrm{m}} \propto K\left(T_{\mathrm{s}}-T_{1}\right)^{-1 / 2}
$$

where $K$ and $T_{1}$ are a proportional constant and the experimental temperature, respectively. $\quad T_{1}$ is kept constant in the present experiment. When gelation occurs at the state of Fig. 8(B), a comparatively large pore structure can be seen (Fig. 7(B)). With an increase of the concentration of PEG, the gelation rate decreased compared with the rate of increase of upper critical solution temperature, and the pore size decreased according to Fig. 8(C) and Eq. (4).

Financial support by a Grant-in-Aid for Scientific Research (C) (No. 05650842) from the Ministry of Education, Science and Culture, Japan, and by the Mazda Foundation for Research, are gratefully acknowledged.

\section{References}

1. H. Mori, H. Yamashita, K. Nakamura and T. Maekawa, J. Ceram. Soc. Jpn., 101, 1180 (1993).

2. H. Komura, K. Nakanishi and N. Soga, Proceedings of Fall Meeting, The Ceramic Society of Japan (1989), p. 238.

3. K. Nakanishi and N. Soga, J. Non-Cryst. Solids, 139, 1(1992).

4. K. Nakanishi and N. Soga, J. Non-Cryst. Solids, 142, 45 (1992).

5. H. Kaji, K. Nakanishi and N. Soga, J. Sol-Gel Sci. Technol., 1, 35 (1993).

6. R. Ota, A. Watanabe, J. Fukunaga and N. Yoshida, $J$. Ceram. Soc. Jpn., 97, 213 (1989).

7. M. Uo, K. Yamashita, M. Suzuki, E. Tamiya, I. Karube and A. Makishima, J. Ceram. Soc. Jpn., 100, 426 (1992).

8. T. Katagiri and T. Maekawa, J. Non-Cryst. Solids, 134, 183 (1991).

9. J. W. Cahn, Acta Metall., 9, 795 (1961).

(Received March 28, 1994)

(Accepted July 11, 1994) 